\title{
Posterior reversible encephalopathy syndrome (PRES) in a patient with complicated appendicitis. A
}

\section{case report}

\begin{abstract}
Posterior reversible encephalopathy syndrome (PRES) is clinical entity characterised by neurologic and radiologic findings. PRES is predominantly caused by malignant hypertension mostly seen in obstetric practise however it has also been associated with sepsis in females. We describe a case of a 11 years old female who presented with complicated appendicitis and developed PRES during her admission at Dr Gearge Mukhari Academic Hospital.
\end{abstract}

Diagnosis: Following exploratory laparotomy for complicated appedicitis with generalized peritonitis, she developed vision loss. CT findings were in keeping with posterior reversible encephalopathy syndrome

Interventions: The patient was managed expectedly for sepsis and shock. Her symptoms resolved and she regained her sight completely with no neurological deficits.

Conclusions: Intra-abominal sepsis is documented risk factor for PRES through inflammatory vascular phenomonon which is poorly understood. Complicated appendicitis can be complicated with this clinical syndrome aspresented in our practise.
Volume 10 Issue 2 - 2020

\author{
M Nyembe, NTshifularo \\ Department of Paediatric Surgery (Sefako Makgatho Health \\ Sciences University), South Africa
}

Correspondence: NTshifularo, Department of Paediatric Surgery (Sefako Makgatho Health Sciences University), South Africa,Email ntshifularo@gmail.com

Received: April 17, 2020 | Published: April 23, 2020

\section{Case report}

We present an 11 year old female who presented to our emergency department transfered from regional hospital with 6 days history of abdominal pain, associated with nausea, vommiting and not passing stools for 3 days. General examination revealed that she was acutely ill and in mild septic shock. Abdominal Xrays revealed distended bowel loops with multiple air fluid levels.

She was resuscitated with crystalloids fluids and antibiotics until endpoints of resuscitations where reached.

On exploratory laparotomy, she had complicated appendicitis with generalised puss contamnination. Appendectomy was done, and abdomen was closed with a percutaneous drain left in. Postoperatively, she was further managed in high care ward.

On day 3 post-operatively, she had single episode of generalised tonic-clonic seizures and she developed loss of vision. This was the first episode of seizure and loss of vision. Opthalmologist examination was normal. Computed Tomography $(\mathrm{CT})$ brain revealed biparietal lobe hypodensities and associated mild surrounding edema with no mass effect. A diagnosis of PRES was made (Figure 1).

After multidiciplinary team inputs, she was started on steriod therapy for central nervous system symptoms. She recovered from her abdominal sepsis and by day 5 post PRES diagnosis she had regained her eyesight and subsequently discharged from hospital after 2 weeks of admission. On 1 week follow-up visit, she had fully recovered from abdominal sepsis and PRES with no neurological deficit.

\section{Discussion}

Posterior reversible encephalopathy syndrome (PRES) first described by Hinchey $\mathrm{J}$ et al. in 1996 is a reversible neurological condition characterized by seizures, impaired consciousness and visual defecit with a typical radiologic findings on computed tomographic (CT) and magnetic resonance imaging (MRI) of brain. ${ }^{1-6}$ There are described risk factors associated with developing this clinical syndrome, typically occurring mostly in females across all ages, ranging from 4-90years of age, ${ }^{3}$ with renal disease, hypertension in pregnancy and as seen in patients on immune suppresive therapies. ${ }^{6} \mathrm{It}$ is also thought to follow a hypovolaemic episode in infection, sepsis, autoimmune disease and electrolyte abnormalities in septic shock. ${ }^{2}$

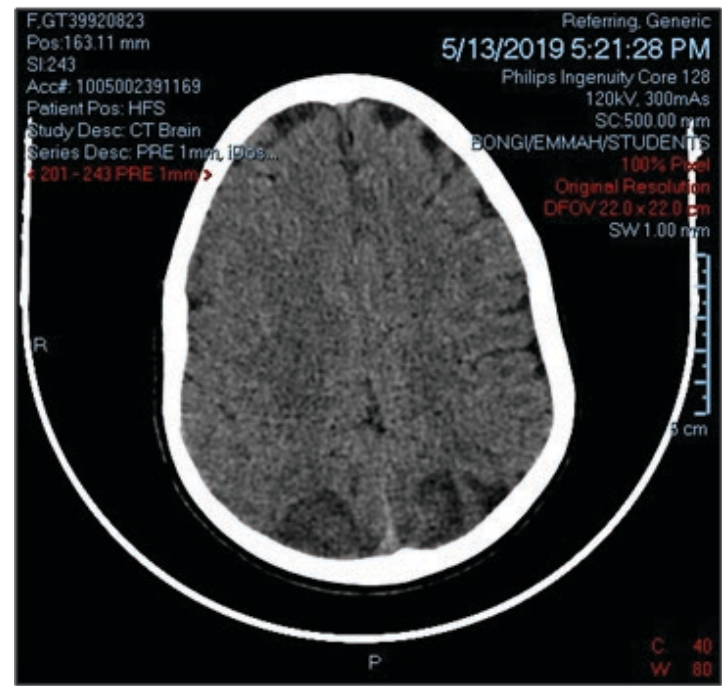

Figure I Biparietal lobe hypodensities and mild edema.

Ourpatient, a young lady with septic shock from complicated appendicitis, peri-operatively developed PRES as evidenced by generalized tonic-clonic seizures and vision lossand typical radiologic pictures. The resolution of the neurological symptoms in this patient, describes the natural history of PRES, which is self limiting and patient 
recovers without permanent neurological deficit. ${ }^{5}$ It is important to exclude organic causes of neurological signs in surgical patients as we did with radiologic imaging.

Pathophysiology still remains unclear. However the most widely accepted theories include the inability of the posterior cerebral circulation to autoregulate in response to acute changes in blood pressure resulting in endothelial dysfunction and vasogenic oedema of the subcortical white matter in the posterior occipital and parietal lobes. ${ }^{4}$

\section{Conclusion}

PRES remains a diagnosis of exclusion as organic pathology must be ruled out before the diagnosis can be made. Clinicians should be aware of this syndrome, typical radiological picture and its self limiting nature. The gold standard for diagnosis of this condition remains a MRI of the brain. ${ }^{2}$ However in a resource constrained setting, diagnosis can also be confirmed with charateristic finding on CT scan as described originally by Hinchey $\mathrm{J}$ et al. ${ }^{1}$

\section{Acknowledgements}

Special thanks to the Sefako Makgatho University Surgical Department Clinical Trail Laboratory for assistance with completing this case report, special thanks to the Department of Peadiatric Surgery for providing oversight on the management of the patient during her hospital stay, the department of Diagnostic \& Interventional Radiology for assistance in radiological diagnosis.

\section{Funding}

None.

\section{Conflict of interest}

The authors have no conflicts of interest to declare.

\section{References}

1. Hinchey J, Chaves C, Appignani B, et al. A reversible posterior leukoencephalopathy syndrome. $N$ Engl J Med. 1996;334(8):494-500.

2. J K Strong, K L Petersen, Hypertension as the trigger for posterior reversible encephalopathy syndrome in paediatric renal patients:An important diagnosis that should not be missed. $S$ Afr Med $J$. 2018;108(4):325-328.

3. Jharendra P Rijal, S Giri, Dawadi S, et al. Posterior reversible encephalopathy syndrome (PRES) in a patient with late postpartum eclampsia. BMJ Case Rep. 2014; pii: bcr2013203162.

4. E Eleonora, M G Piccioni, Schiavi MC, et al. Postpartum posterior reversible encephalopathy syndrome (PRES): three case reports \& litrature review. Case Rep Obstet \& Gynecol. 2019:9527632.

5. Aulakh P, Fatakhov E, Koch CF, et al. Posterior reversible encephalopathy syndrome with documented hyponatraemia; Case Report. BMJ Case Rep. 2013.

6. Cherniawsky H, Merchant N, Sawyer M, et al. A case report of posterior reversible encephalopathy syndrome in a patient receiving gemcitabine and cisplatin. Medicine (Baltimore). 96(8):e5850. 